Proceedings of the 2004 IEEE

lnternational Conference on Networking, Sensing \& Control

Taipei, Taiwan, March 21-23, 2004

\title{
A design of small-area automatic wheelchair
}

\author{
Chuan-heng Hsiao, Ming-long Lee, Yuan-Chung Shen, and Feipei Lai \\ Department of Computer Science and Information Engineering \\ National Taiwan University, Taipei, Taiwan \\ E-mail: chshou@csie.ntu.edu.tw, b9901066@ee.ntu.edu.tw, \\ b89040@csie.ntu.edu.tw, flai@csie.ntu.edu.tw
}

\begin{abstract}
There are lots of handicaps and elders in the world. Many of them are not able to move as easily as normal people. It will be very useful if we develop an automatic wheelchair to help them move more freely. In this paper we will propose a design of small-area automatic wheelchair to help handicaps or elders be able to move easily in a small area. The most concern in this project is low cost with acceptable performance rather than high velocity or high accuracy. The design integrates several technologies to apply on the wheelchair, including wireless positioning, automatic mobile technology, and wireless communication. On the other hand, we propose a design that duplicable information can be computed on server side, and information which is not duplicable can be computed in constant time. Therefore, our design of small-area automatic wheelchair can cost very low. We will also propose a design of building map information to make the whole design more complete.
\end{abstract}

\section{Introduction}

It is important for everyone to move easily. However, some people have difficulty to do so. Some of these people may be blinds. They may depend on other more sensitive sensors and guiding dogs or guiding sticks heavily to move. Some of these people may have problems in their legs. These people may need wheelchairs or crutches to move. Some of these people may be too old to move. They may also need wheelchairs to move.

In the past, a wheelchair is powered with human hands, and it is very difficult to move more than 100 meters. Currently there is a kind of electric wheelchair, which improves this situation significantly. However, it still needs people to watch out to avoid accident. The energy to watch out accidents should be saved, and some elders or handicaps may even not be able to do so. It is very inconvenient for these people in some small areas, such as campus, zoo, museum, or some factories. Therefore, it will help these people significantly if we have a small-area automatic wheelchair.

There are at least three advantages of a small-area automatic wheelchair compared with traditional autonomous vehicles on road or on highway. These advan- tages make it possible to be practical and useful widely. Three advantages are listed as the following:

- Complexity: In normal situations, such as transportation in a city, it may need to deal with more than hundred thousands places, thousands of routes, and millious of cars. The complexity in normal situations is very high. However, in these small areas, it just needs to deal with less than thousands of places. Therefore, the complexity in these areas can be reduced significantly.

- Velocity: In normal situations, such as cars on roads. Vehicles usually move with more than 40 $\mathrm{KM} / \mathrm{hr}$. However, $5 \mathrm{KM} / \mathrm{hr}$, or even $3 \mathrm{KM} / \mathrm{hr}$ is enough for handicaps in these small areas.

- Safety: In normal situations, the damage is very serious if some accident happened. However, because the velocity in these areas is very low, the damage will not be serious if some accident happened.

The design of small-area automatic wheelchair can be viewed as an application of light-weight automatic transportation system. Light-weight automatic transportation system can be defined as the following:

- Light-weight automatic transportation system: Light-weight automatic transportation system is a kind of automatic transportation system which has some characteristics as the following:

- The number of nodes $n$ on the maps is less than some constant value $C_{n}$.

- The velocity $v$ of vehicle is less than some constant value $C_{v}$.

With current technology, $C_{n}$ is set as 1000 and $C_{v}$ is set as $5 \mathrm{KM} / \mathrm{hr}$ in this paper. Light-weight automatic transportation system can be useful in a small area and non-efficient transportation. This transportation system is similar to Kelly's AMTS [1], which transports materials in factories.

Since usually the handicaps or elders are not very rich because of the inability of movement, the most concern 
in this project is not the velocity or accuracy but low cost with acceptable performance. This is slightly different with many other autonomous vehicle projects.

Applying automatic vehicles on wheelchair has been proposed by Madarasz in 1986 [2]. However, technology has been improved significantly during these years. Zhao proposed using wireless positioning onto intelligent transportation system in 2000 [3]. Grzywna proposed a modulation of building automatic vehicles in 2001 [4]. Also, some technologies of other projects are similar to part of the technologies we need [5] [6]. We integrate these technologies to have a complete design of smallarea automatic wheelchair.

\section{Designation}

\subsection{Concept}

Here we try to apply agent concept onto the smallarea autonomous wheelchair. As mentioned by Russeull and Norvig [7], agent can be viewed as Figure 1.

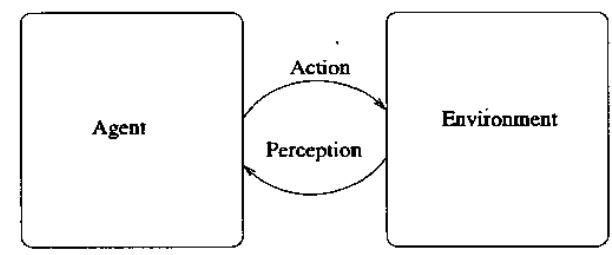

Figure 1: Diagram of agent concept

As shown on Figure 1, the agent constantly gets necessary information from environment and then acts and affects back to the environment. The information is modulated as at least four necessary parts for a smallarea autonomous wheelchair:

- map of the area: The map contains the information of routes, places we can go, buildings and other obstacles. This information provides the whole view of the small area.

- shortest route: Although this can be computed after retrieving the map, since the time complexity of computing the shortest route is $O\left(n^{3}\right)$ [8], it will be efficient if it can be provided by the others.

- positioning: The wheelchair needs to know where it is currently, and then it can decide how to move to the target entry.

- sensing obstacles: Because the environment is very dynamic, there must be some minor obstacles not listed on the map. The wheelchair must have the ability to sense these obstacles to avoid accidents.

The actions are also modulated as four parts for smallarea autonomous wheelchair, forward, backward, leftturn and right-turn.

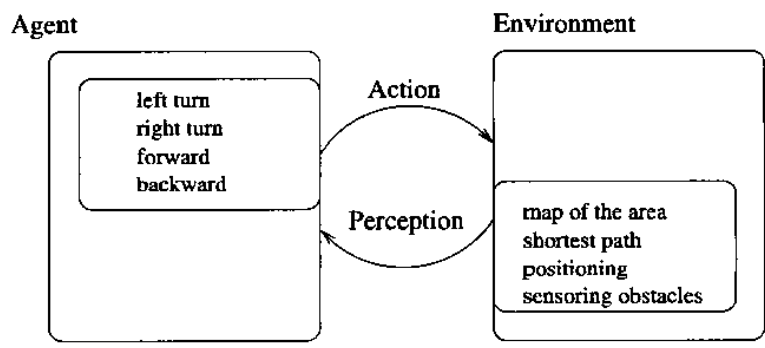

Figure 2: Diagram of small-area autonomous wheelchair

Therefore, the diagram of our design of small-area autonomous wheelchair is shown as Figure 2.

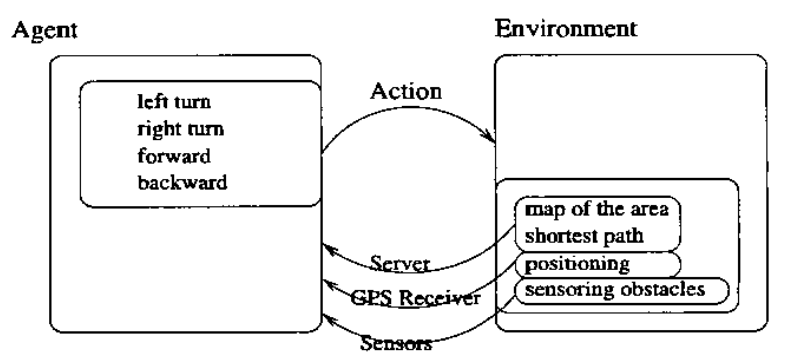

Figure 3: Diagram of methods of retrieving information

Map information and the shortest route information are duplicable for every small-area autonomous wheelchairs in the same area. Therefore, they can be served with the server in the area. The methods of retrieving information is illustrated as Figure 3.

The bottleneck of computation is in the shortest route, which is $O\left(n^{3}\right)$ [8]. The rest of data can be computed in $O(1)$ after data is retrieved. Therefore, our small-area autonomous wheelchair can be equipped with a lightweight mobile device, such as PDA, mobile phone, or Intel 8051 microprocessor products.

\subsection{Procedure}

The application of a small-area autonomous wheelchair can be completed with the following procedure:

- In server site, the location of each node on roads and target places can be retrieved by using wireless positioning technology. And then the information is recorded on the map. After building this map, the server can compute the shortest route with time complexity $O\left(n^{3}\right)$.

- When a small-area autonomous wheelchair entering into the area, the mobile device built in the wheelchair will automatically communicate with the server of the small area, and get the map of the area. 
- After getting the map of the small area, user can choose one of the places listed on the map. with various input method, such as keyboard, mouse, or even speech recognition. And then the smallarea automatic wheelchair will send request of the shortest route to the server.

- The server will send back the shortest route. And then the small-area automatic wheelchair will go to the target place according to the route. The route between nodes on the road is assumed to be straight when building the map. Also, the small-area automatic wheelchair can use wireless positioning to get the information of current location.

- With current position $P_{c}$ and the next node we will pass $P_{n}$, the small-area automatic wheelchair can compute the direction $D_{n}$ according to the following equation:

$$
D_{n}=P_{n}-P_{c}
$$

- The current direction of the small-area automatic wheelchair $D_{c}$ can be iterated by having the head and tail of wheelchair with wireless positioning devices. The difference of direction $D$ can be computed as the following:

$$
D=D_{n}-D_{c}
$$

- $D$ can be expressed as a two-dimensional vector $\left(X_{D}, Y_{D}\right)$. Therefore, the radius $\theta$ to be turned can be computed as the following:

$$
\begin{aligned}
\theta & =\sin ^{-1}\left(\frac{Y_{D}}{\sqrt{X_{D}^{2}+Y_{D}^{2}}}\right) \\
& =\cos ^{-1}\left(\frac{X_{D}}{\sqrt{X_{D}^{2}+Y_{D}^{2}}}\right)
\end{aligned}
$$

\subsection{A Problem of the Procedure}

There is a situation that the sensors detect some obstacle on the place where the small-area automatic wheelchair will go. A simple solution is dealt with the following procedure:

1. First, it is assumed that the obstacle is movable. Therefore, the small-area automatic wheelchair will wait for some short time $t$ and then try to move again.

2. If the obstacle is still there, then it is assumed that the obstacle is not large enough to block the whole road. Therefore, the small-area automatic wheelchair will try to move to other directions for some short distance and then back to the target places.

3 . If the small-area automatic wheelchair continuely meets the obstacle for some time $T$, it assumes that this road is blocked, and sends the request to server to reiterate another route.

\subsection{Map Building}

Many projects are similar to this paper. However, one problem that these kinds of project can not be useful is that these projects do not mention how to provide the map for these projects. Because our positioning is based on wireless positioning, the map of the small area can be got from local government. If the is not available from government, the data can be retrieved with the following procedure:

- Choose the key nodes which need to be positioned.

- Use wireless positioning on these nodes.

- Complete the map by grouping nodes as buildings, routes, entries, or obstacles.

\section{Implementation}

A prototype is implemented with the following specification:

\subsection{Equipment}

The equipment is illustrated as the following:

- wheelchair: We use electric 3-wheel scooter produced by PiHsiang Machinery MFG. Co. as our wheelchair, which is shown in Figure 4.

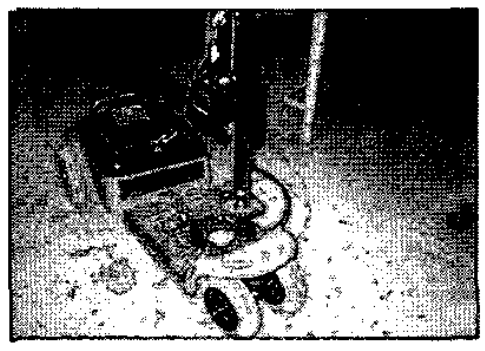

Figure 4: A motor and a gear added on the scooter

- wireless positioning: We use the DGPS receiver produced by Holux Technology Inc. as shown in Figure 5.

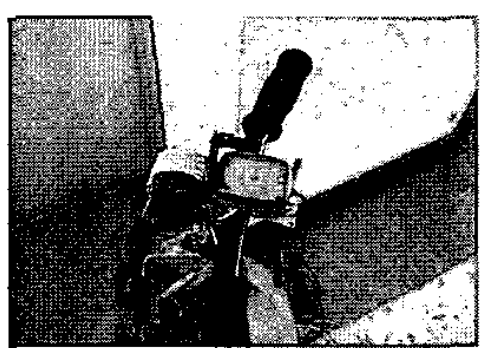

Figure 5: DGPS Receiver 
- mobile controlling: Although the electric scooter has the power to move forward and backward, the left turn and the right turn need to be manipulated manually. Therefore, we add a gear and a motor with $5 \mathrm{~V} / 12 \mathrm{~A}$ to implement the left turn and right turn, which is shown as Figure 4. We use Intel 8051 microprocessor to control the movement of the wheelchair, which is shown as Figure 6 .

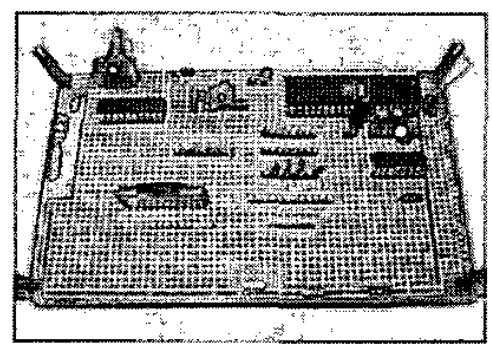

Figure 6: Automatic controlling module with 8051

- map building: We develop a program to build map with wireless positioning, which is shown as Figure 7.

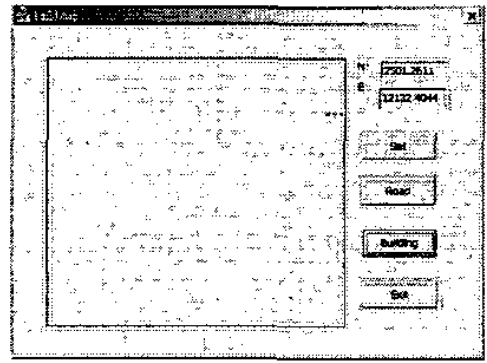

Figure 7: User interface of building map

- main program: We develop a program to implement the procedure of our designation of small-area automatic wheelchair.

\subsection{Cost}

The cost of the implementation can be illustrated as Table 1. The price is less than $\$ 1000$, which is affordable by many people.

\subsection{Performance}

We tested the prototype in our campus. The speed of wheelchair is set to be $4 \mathrm{KM} / \mathrm{hr}$, which is similar to the speed of walk with normal people. The error of DGPS receiver is around $4 \mathrm{M}$ with this speed. Since the width of roads in our campus is about $5 \mathrm{M}$, the error is tolerable. Each step is completed in 1s. Each step moves $2 \mathrm{M}$. Figure 8 is the track of the small-area automatic
Table 1: The cost of the implementation

\begin{tabular}{|c|c|}
\hline software & $\$ 0$ \\
wheelchair & $\$ 800$ \\
DGPS receiver & $\$ 100$ \\
motor & $\$ 30$ \\
gear & $\$ 5$ \\
8051 module & $\$ 30$ \\
\hline total & $<\$ 1000$ \\
\hline
\end{tabular}

wheelchair on a straight $35 \mathrm{M}$ road, where bold line is ideal route, and dash line is real route of the small-area automatic wheelchair passing the road. Although the performance shown in Figure 8 is not very good, it is acceptable in this test.

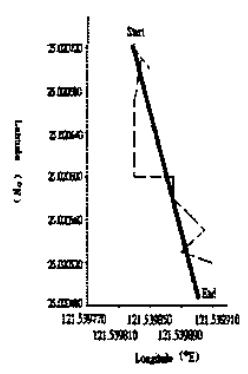

Figure 8: Track of small-area automatic wheelchair on $35 \mathrm{M}$ straight road

\section{Conclusion}

It is very useful to help handicaps or elders to move easily in a small area. We integrate several technologies to propose a complete design of small-area automatic wheelchair. The whole system is modulated as several parts which can be improved independently. Wireless positioning is used to replace traditional role of computer vision in automatic vehicle. Wireless communication is used to retrieve map and the shortest route information from local server. We also provide a prototype implementation. The cost is low and the performance is acceptable, which fits our concern.

\section{Future Work}

Here we have a prototype of small-area automatic wheelchair. However, the tradeoff of cost and performance should be tuned further to find the most suitable combination.

On the other hand, hierarchical map structure may be able to make the designation extended to a large area. This designation is to view map of a large area with 
major route first, and then we zoom in the map with small area and find the exact entry.

\section{References}

[1] A. Kelly and A. T. Stentz, "An approach to rough terrain autonomous mobility," in International Conference on Mobile Planetary Robots, Jan. 1997.

[2] R. L. Madarasz, L. C. Heiny, R. F. Cromp, and N. M. Mazur, "The design of an autonomous vehicle for the disabled," IEEE Journal of Robotics and Automation, vol. 2, pp. 117-126, 1986.

[3] Y. Zhao, "Mobile phone location determination and its impact on intelligent transportation systems," IEEE Trans. on Intelligent Transportation Systems, vol. 1, pp. 55-64, 2000.

[4] J. W. Grzywna, D. E. M. Schwartz, and D. A. A. Arroyo, "Kelvin: Kart for long distance vision navigation," in 2001 Florida Conference on Recent Advances in Robotics, Florida A\&M University, May 10-11 2001.

[5] A. Zelinsky and S. Yuta, "A unified approach to planning, sensing and navigation for mobile robots," in International Symposium on Experimental Robotics (3rd ISER), Kyoto, Japan. Oct. 1993, pp. 444-455.

[6] A. A. Reyes and A. Dogan, "Autonomous vehicle lab initiative at uta," in AIAA Unmanned Unlimited Systems, Technologies, and Operations-Acrospace, Land, and Sea Conference and Workshop \& Exhibit, Hyatt Regency Islandia, San Diego, California, Sept. 15-18, 2003.

[7] S. Russell and P. Norvig, Artificial Intelligent $A$ Modern Approach, 2nd ed. Prentice Hall, 2003.

[8] T. H. Cormen, C. E. Leiserson, and R. L. Rivest, Introduction to Algorithms. Cambridge, Massachusetts: MIT Press, 1989, ch. 26. 